\title{
Alterations in drug metabolizing activities in acute hepatosteatosis induced by intake of a high-carbohydrate/fat-free diet after food deprivation
}

\section{Chul Won Ahn', Jung Ah Lee', Jae Hak Park², Young Chul Kim}

${ }^{1}$ College of Pharmacy, Seoul National University, San 56-1 Shinrim-Dong, Kwanak-Ku, Seoul, Korea; ${ }^{2}$ College of Veterinary Medicine, Seoul National University, San 56-1 Shinrim-Dong, Kwanak-Ku, Seoul, Korea

Corresponding author: Young C. Kim, Professor of Toxicology, College of Pharmacy, Seoul National University, San 56-1 Shinrim-Dong, Kwanak-Ku, Seoul 151-742, Korea.

Submission date: December 23, 2015, Acceptance date: January 29, 2016: Publication date: January 30, 2016

\begin{abstract}
Background: Lipid accumulation in hepatocytes constitutes a major component in the pathogenesis of chronic liver injury. However, the impact of lipid deposition in liver on drug metabolizing capacity remains unclear.
\end{abstract}

Purpose of the study: The present study was undertaken to evaluate the changes in hepatic cytochrome P-450 (CYP) enzymes in acute hepatosteatosis.

Methods: The rat subjects fasted for $48 \mathrm{~h}$, and then were provided with a highcarbohydrate/fat-free diet $(\mathrm{FH})$ or a normal diet $(\mathrm{FN})$ for $48 \mathrm{~h}$.

Results: Hepatic lipid accumulation was significant in the FH group compared to the FN group. In the FN group, there was a small increase in microsomal $p$-nitrophenol hydroxylase, $p$ nitroanisole $O$-demethylase, and erythromycin $N$-demethylase activities. In contrast, aminopyrine $N$-demethylase activity significantly decreased. However, the microsomal enzyme activities were all inhibited by FH intake. Immunoblotting analysis revealed that CYP2E1, CYP1A, CYP3A, and CYP2B1/2 proteins decreased in the $\mathrm{FH}$ group. Expression of corresponding CYP mRNAs was also down-regulated. A dose of $\mathrm{CCl}_{4}$, a CYP2E1 substrate, was administered to rats fed with different diets. The liver injury was significantly lower in the FH 
group compared to the FN group, as determined by the elevation of serum enzyme activities and histopathological examination.

Conclusions: The results revealed that acute hepatosteatosis may result in a significant alteration in hepatic CYP-mediated metabolizing capacity, which can precipitate erratic responses of liver to various endogenous and exogenous substances.

Key words: hepatosteatosis, cytochrome $\mathrm{P} 450$, non-alcoholic fatty liver disease, drug metabolizing capacity

\section{INTRODUCTION}

Hepatosteatosis, or fatty liver, is the most common histopathological change in liver disorders. Fatty liver can result from ingestion of alcohol and certain drugs, including corticosteroids, some antibiotics and nonsteroidal anti-inflammatory agents [1]. Additionally, because of its high prevalence in obesity, diabetes and insulin resistance, non-alcoholic fatty liver disease (NAFLD) is increasingly appreciated as a hepatic manifestation of the metabolic syndrome [2,3]. It is suspected that the accumulation of lipid in liver, regardless of its etiology, would modulate the expression and activity of various drug metabolizing enzymes. The metabolic deposition of drugs and chemical substances may then be influenced due to the incompetency of hepatic drug metabolizing capacity, resulting in altered pharmacological efficacy or adverse drug reactions. In fact, changes in pharmacokinetics of various drugs were seen within obese patients $[4,5]$.

$\mathrm{CCl}_{4}$ is a model hepatotoxicant for free radical-induced liver injury. $\mathrm{CCl}_{4}$ is bioactivated to trichloromethyl free radical via CYP-dependent reductive dehalogenation, in which CYP2E1 plays a major role [6-8]. The trichloromethyl free radical binds covalently to lipids and proteins, resulting in membrane damage and inhibition of protein functions. Moreover, this radical attacks polyunsaturated fatty acids, generating organic free radicals which may react with oxygen to form peroxides and other cytotoxic metabolites [9]. Therefore, it is accepted that CYP2E1 activity is a key contributing factor in initiating the $\mathrm{CCl}_{4}$-induced liver toxicity.

Earlier studies showed that liver fatty acid synthesis is enhanced by dietary carbohydrates after short term fasting. For example, within rats who were fed a high-carbohydrate diet after being deprived of food for 2 days, the lipid synthesis and triglyceride levels in the liver were increased [10]. Both circulating and de novo synthesized fatty acids in liver were implicated in 
the disruption of hepatic lipid homeostasis, leading to lipid accumulation in the livers of the rats who fasted before being introduced to a high-carbohydrate/fat-free diet [11]. In this study, we examined the changes in the CYP enzyme system and $\mathrm{CCl}_{4}$ toxicity in acute hepatosteatosis. Since acute fatty liver induced by feeding rats with a high-carbohydrate/fat-free diet after food deprivation may avoid other complications associated with the progression of chronic liver injury, it is anticipated that the use of this model can reveal a clearer picture of the consequences of fat accumulation in liver.

\section{METHODS}

Animals and treatments: Male Wistar rats, weighing 200-220 g, were purchased from OrientBio Experimental Animals (Seongnam, Korea). The use of animals was in compliance with the guidelines established by the Animal Care Committee of this institute. Rats were acclimated to temperature $\left(22 \pm 2^{\circ} \mathrm{C}\right)$ and humidity $(55 \pm 5 \%)$ controlled rooms with a 12-h light/dark cycle (light: 0700-1900, dark: 1900-0700) for 1 wk before use. Rats were provided with a standard regular diet (Cargill Agri Purina, Seongnam, Korea) before food deprivation for $48 \mathrm{~h}$. After the fasting period rats were fed a regular diet $(\mathrm{FN})$ or a high-carbohydrate/fat-free diet (FH; G-Bio, Gwachon, Korea) for $48 \mathrm{~h}$ until sacrifice for measurement of the CYP enzyme system. Different groups of rats were challenged with a dose of $\mathrm{CCl}_{4}(4 \mathrm{mmol} / \mathrm{kg}$, ip) at $48 \mathrm{~h}$ after refeeding the rats for measurement of liver injury. The standard regular diet contained $64.1 \%$ carbohydrate, $20 \%$ protein, $6 \%$ fiber, $4.5 \%$ fat with appropriate mineral salts and vitamins. The highcarbohydrate/fat free diet was made up of $80 \%$ carbohydrate (sucrose and corn starch, 1:1), $16 \%$ protein, $0 \%$ fat, in addition to appropriate mineral salts and vitamins.

Measurements of liver injury: The hepatotoxicity of $\mathrm{CCl}_{4}$ was estimated by elevation of serum enzyme activities. At $24 \mathrm{~h}$ after $\mathrm{CCl}_{4}$ treatment, blood was sampled from abdominal aorta in rats under light ether anesthesia. Serum sorbitol dehydrogenase (SDH) was determined spectrophotometrically [12]. Aspartate aminotransferase (AST) and alanine aminotransferase (ALT) activities were determined using the method of Reitman and Frankel [13]. Thiobarbituric acid reactive substances (TBARS) were measured to quantify lipid peroxidation in liver [14].

For evaluation of lipid accumulation in liver, sections of frozen liver were sliced at $10 \mu \mathrm{m}$, immersed in propylene glycol for $5 \mathrm{~min}$, and stained with Oil red $\mathrm{O}$ for $7 \mathrm{~min}$. After rinsing with $85 \%$ propylene glycol and distilled water, the sections were counterstained with hematoxylin for 
2 min before microscopic examination. For histopathologic assessment of liver injury, the left lateral lobe of liver was sliced and fixed in $10 \%$ neutral-buffered formalin. Tissues were processed routinely and embedded in paraffin. Sections of 2-3 $\mu \mathrm{m}$ in thickness were subjected to hematoxylin and eosin staining before microscopic examination.

Changes in CYP activities: Liver was homogenized in an ice-cold buffer of $0.154 \mathrm{M} \mathrm{KCl} / 50$ $\mathrm{mM}$ Tris- $\mathrm{HCl}$ with $1 \mathrm{mM}$ EDTA, $\mathrm{pH}$ 7.4. The homogenate was centrifuged at $10000 \mathrm{~g}$ for $20 \mathrm{~min}$, and the supernatant was further centrifuged at $104000 \mathrm{~g}$ for $60 \mathrm{~min}$. The microsomal pellet was suspended in the homogenizing medium, followed by recentrifugation at $104000 \mathrm{~g}$ for $60 \mathrm{~min}$. The final pellet obtained was resuspended and used for measurement of microsomal CYP activities. Hepatic microsomal CYP activities were determined using the method described elsewhere [15]. $P$-nitrophenol hydroxylase activity was determined by measuring the formation of $p$-nitrocatechol. Reaction mixtures (final volume of $1 \mathrm{ml}$ ) contained $0.1 \mathrm{mM} p$-nitrophenol, $1.0 \mathrm{mM}$ ascorbic acid, $0.1 \mathrm{ml}$ of microsomal suspension and $1 \mathrm{mM}$ NADPH in $0.1 \mathrm{M}$ potassium phosphate buffer ( $\mathrm{pH}$ 6.8). Incubation was conducted at $37^{\circ} \mathrm{C}$ for $3 \mathrm{~min}$. Aminopyrine $\mathrm{N}$ demethylase, erythromycin $N$-demethylase and $p$-nitroanisole $O$-demethylase activities were determined by measuring the production of formaldehyde or $p$-nitrophenol. Reaction mixtures consisted of $0.1 \mathrm{ml}$ microsomal suspension, $1 \mathrm{mM} \mathrm{NADPH}$, the substrate $(5 \mathrm{mM}$ for aminopyrine or erythromycin; $0.1 \mathrm{mM}$ for $p$-nitroanisole) and $0.1 \mathrm{M}$ potassium phosphate buffer (pH 7.4), in a total volume of $1.0 \mathrm{ml}$. Incubation was conducted at $37^{\circ} \mathrm{C}$ for $10 \mathrm{~min}$, before the addition of cold $20 \%$ trichloroacetic acid to stop the reactions.

Immunoblotting and gene expression analysis: Liver microsomal protein $(20 \mu \mathrm{g})$ was loaded, separated by gel electrophoresis, and transferred to nitrocellulose membranes by electroblotting. The membranes were blocked in Tris-buffered saline containing $5 \%$ nonfat dry milk and $0.1 \%$ Tween-20. The blots were incubated with primary antibodies at $4^{\circ} \mathrm{C}$ overnight. Secondary antibodies were then conjugated to horseradish peroxidase at room temperature for $1 \mathrm{~h}$. Monoclonal antibodies against rat CYP1A1/2, CYP2B1/2, and polyclonal antibodies against CYP2E1, CYP3A (Detroit R\&D, Detroit, MI) were used as probes. Blots were detected by enhanced chemiluminescence. Signal intensity was normalized relative to that of $\beta$-actin.

Gene expression was examined by quantitative real-time PCR. Total hepatic RNA was isolated using TRIzol ${ }^{\circledR}$ reagent (Invitrogen, Carlsbad, CA) and purified by an RNeasy Mini Kit 
(Qiagen, Hilden, Germany). cDNA was obtained from isolated RNA template using reverse transcription. Gene expression was measured using cDNA with corresponding gene-specific primers and a Light-Cycler System (Roche Diagnostic, Mannheim, Germany), following the manufacturer's standard protocol. The relative expression was normalized to that of glyceraldehyde-3-phospate dehydrogenase (GADPH). The gene-specific primers applied were as follows: CYP2E1 sense, 5'-TCTGAGATATGGGCTCCTGA-3', anti-sense, 5'CGGGGAATGACACAGAGTTT-3'; CYP3A1 sense, 5'-AGAAATTCGATGTGGAGTGC-3', anti-sense, 5'-AGGTTTGCCTTTCTCTTGCC-3'; CYP3A2 sense, 5'-GCTTTCAGC TC TCACACTGGAA-3', anti-sense, 5'-TCTATGGGTTCCAAGTCGGTAGA-3'; CYP1A2 sense, 5'-GGGTGTTGAGAGGCACAAGG-3', anti-sense, 5'-CACTAGGGCCTGCTTGAT GG-3'; CYP2B1/2 sense, 5'-CCCAATGTTTGGTGGAGGAA-3', anti-sense, 5'-CTGTGAT GCACTGGAAGAGGAA-3'; GAPDH sense, 5'-GACCCCTTCATTGACCTCAAC-3', antisense, 5'-GGAGATGATGACCCTTTTGGC-3'.

Statistical analysis: All results were expressed as the mean \pm SEM. Means of the different diet groups were compared using one-way ANOVA followed by Newman-Keuls multiple range test. The acceptable level of significance was established at $\mathrm{P}<0.05$.

\section{RESULTS AND DISCUSSION}

It has been known that diet composition following food deprivation independently manipulates hepatic fat content in rats $[16,17]$. In particular, the feeding of a high carbohydrate/fat-free diet after starvation in rats was demonstrated to result in the sequential accumulation of triglyceride in their liver tissue. $6 \mathrm{~h}$ after refeeding, a slight microvacuolar steatosis, mainly localized in zone I, was followed afterwards by microvacuolar steatosis, which extended to all three zones of hepatic lobules [11]. Within that study, the authors proposed that fasting-refeeding a high carbohydrate/fat-free diet could constitute a good model of dietary-induced steatosis without apparent necroinflammation. The present study, which utilized the same fasting-feeding schedule, revealed that triglyceride accumulation in liver increased significantly within the rats fed FH after a 2 day fasting when compared with the rats fed FN after fasting (Fig. 1A). Histopathological examination of liver samples also demonstrated similar results (Fig. 1B). Lipid accumulation in liver was markedly increased in the FH group, when examined after Oil red O staining. But there appeared to be no significant inflammatory reactions in the liver of rats fed 
FH or FN after a 2 day food deprivation. The histological changes observed in the rats fed FH after food deprivation suggest that this rapid dietary manipulation could serve for a suitable model of dietary-induced acute steatosis.
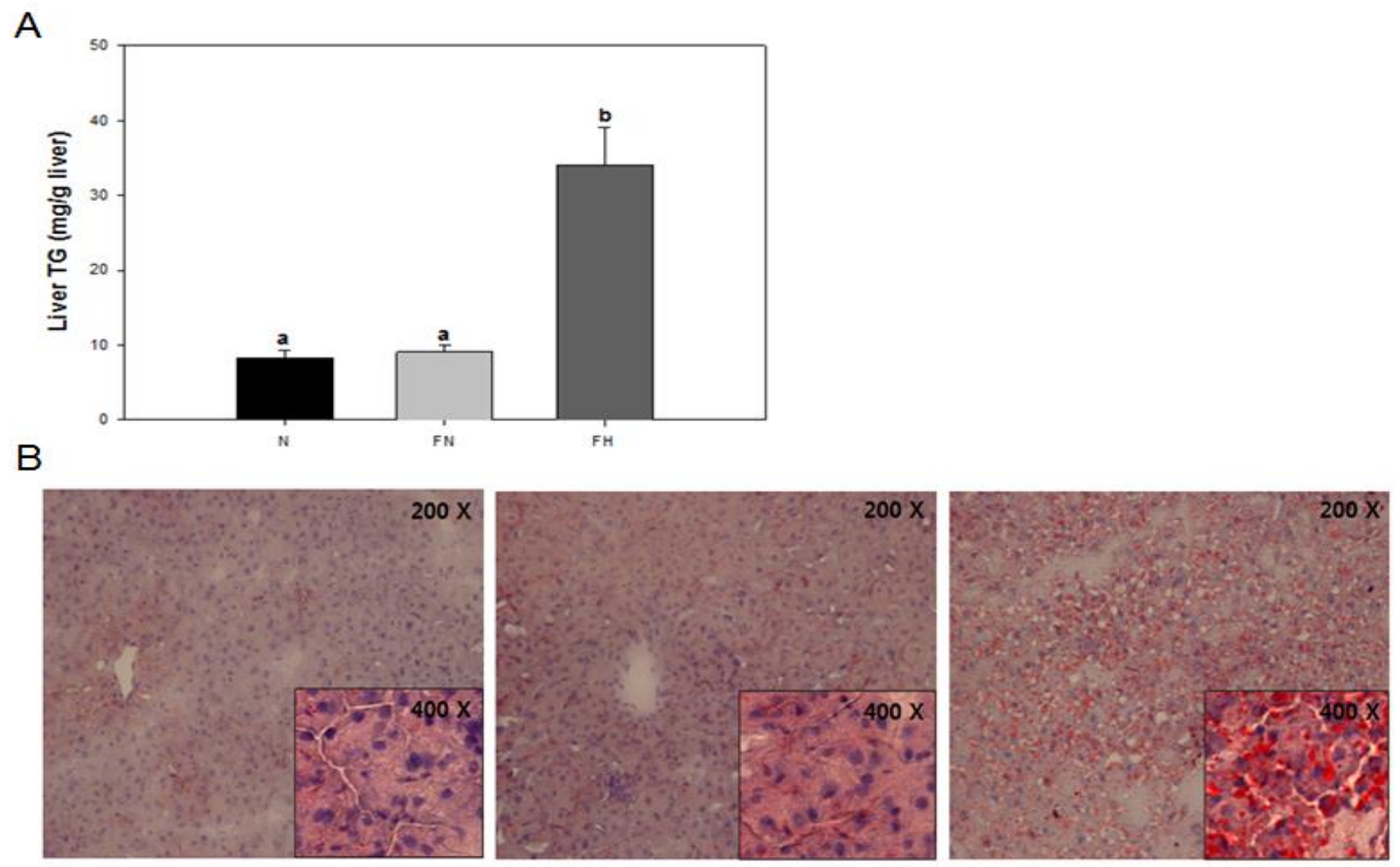

Fig. 1. Lipid accumulation in rat liver. (A) Hepatic triglyceride levels in rats. Each Value represents the mean \pm SE for 6 or 7 rats. Values with different letters $(a, b)$ are significantly different one from another (one-way ANOVA followed by Newman-Keuls multiple range test, $\mathrm{P}<0.05$ ). (B) The liver samples were stained with Oil red $\mathrm{O}$ for microscopic examination. $\mathrm{N}$, normal control; FN, refeeding a regular diet after fasting; FH, refeeding a high carbohydrate/fat-free diet after fasting.

Two days of fasting resulted in a small change in the CYP activities in liver (Table 1). Activities of $p$-nitrophenol hydroxylase, $p$-nitroanisole demethylase, and erythromycin $N$ demethylase were induced slightly, significantly within the rats who fed a normal diet after fasting (FN group), rather than the rats who were fed a normal diet without feeding interruption. In contrast, aminopyrine $\mathrm{N}$-demethylase activity was decreased in the FN group compared to the group who had regular feeding. However, intake of FH after fasting significantly inhibited all the CYP enzyme activities determined in this study. The results of immunoblotting analysis showed that expression of CYP proteins was not affected by FN intake after fasting, except for CYP1A which was decreased slightly (Fig. 2). Nonetheless, in the FH group, the expression of CYP2E1, CYP3A, CYP1A, and CYP2B1/2 proteins was significantly lower than that found in the FN 
group. Similar changes were shown in the CYP mRNA expression (Fig. 3). Deprivation of food for 2 days followed by FN intake did not affect the expression of CYP2E1, CYP3A2, CYP1A2, or CYP2B1/2 mRNAs, but decreased that of CYP3A1 mRNA. FH intake after fasting depressed the expression of all the CYP mRNAs measured markedly.

Table 1. Relative enzyme activities of CYP isoforms in the liver of rats after feeding of experimental diets

\begin{tabular}{crrrr}
\hline & $\begin{array}{c}p \text {-Nitrophenol } \\
\text { Hydroxylase }\end{array}$ & $\begin{array}{c}p \text {-Nitroanisole } \\
\text { Demethylase }\end{array}$ & $\begin{array}{c}\text { Erythromycine } \\
N \text {-Demethylase }\end{array}$ & $\begin{array}{c}\text { Aminopyrine } \\
N \text {-Demethylase }\end{array}$ \\
\cline { 2 - 5 } & & \multicolumn{2}{c}{$($ nmoles/min/g liver $)$} \\
\hline N & $9.17 \pm 0.35^{\mathrm{a}}$ & $10.52 \pm 0.43^{\mathrm{a}}$ & $59.58 \pm 4.03^{\mathrm{a}}$ & $46.22 \pm 3.60^{\mathrm{a}}$ \\
$\mathrm{FN}$ & $12.32 \pm 0.47^{\mathrm{b}}$ & $13.54 \pm 0.91^{\mathrm{b}}$ & $72.49 \pm 4.67^{\mathrm{b}}$ & $34.45 \pm 1.88^{\mathrm{b}}$ \\
FH & $7.61 \pm 1.01^{\mathrm{a}}$ & $8.79 \pm 0.57^{\mathrm{a}}$ & $40.21 \pm 3.76^{\mathrm{c}}$ & $29.12 \pm 2.84^{\mathrm{b}}$ \\
\hline
\end{tabular}

Each Value represents the mean \pm SE for 6 or 7 rats. Values with different letters $(a, b, c)$ are significantly different one from another (one-way ANOVA followed by Newman-Keuls multiple range test, $\mathrm{P}<0.05)$.
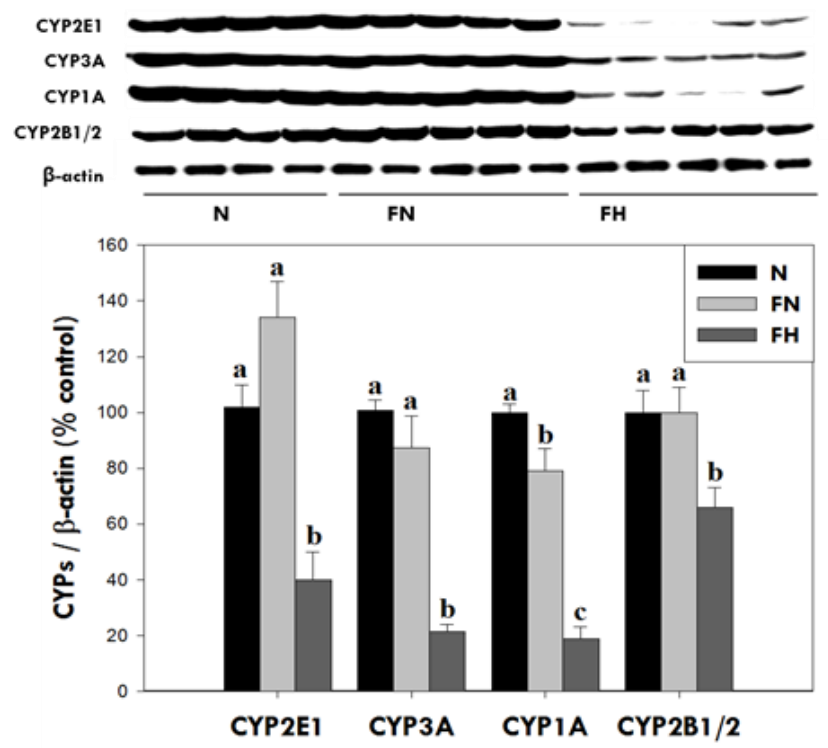

Fig. 2. Expression of CYP proteins in liver. Values with different letters $(\mathrm{a}, \mathrm{b}, \mathrm{c})$ are significantly different one from another (oneway ANOVA followed by Newman-Keuls multiple range test, $\mathrm{P}<0.05$ ).

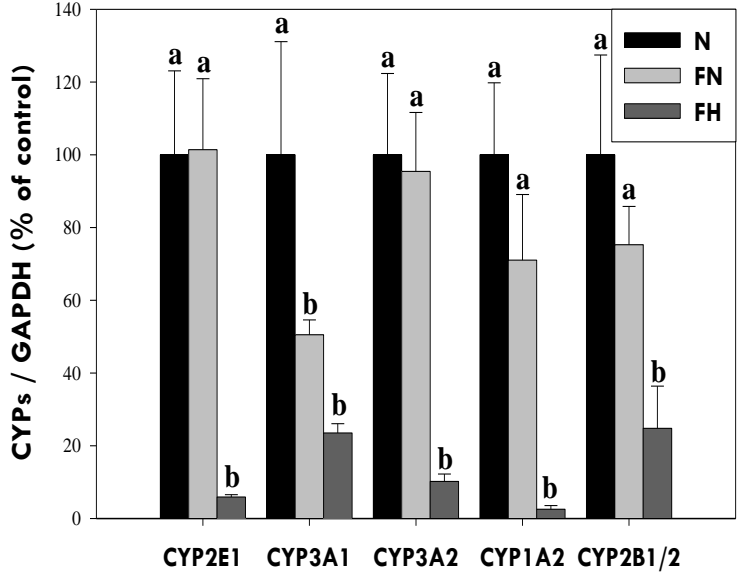

Fig. 3. Expression of CYP mRNA in liver. Each value represents the mean \pm SE for 6 or 7 rats. Values with different letters $(a, b)$ are significantly different one from another (one-way ANOVA followed by NewmanKeuls multiple range test, $\mathrm{P}<0.05$ ). 


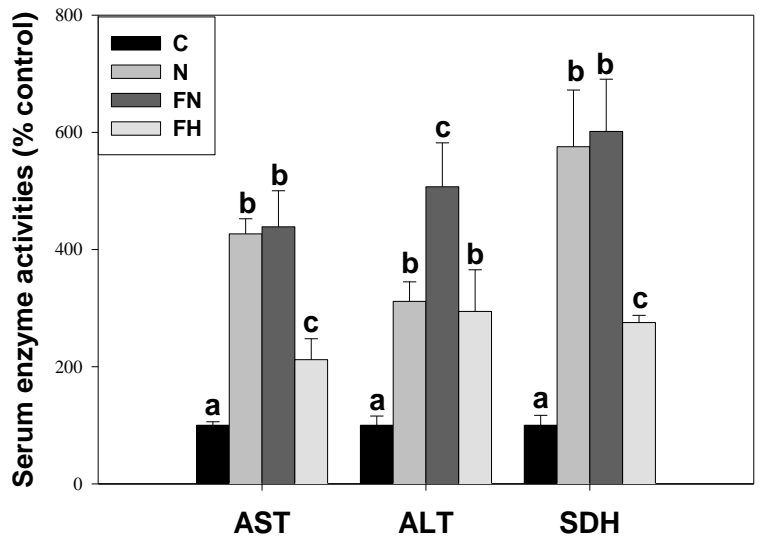

Fig. 4. Elevation of serum enzyme activities after $\mathrm{CCl}_{4}$ administration. Each Value represents the mean \pm SE for 5 rats. Values with different letters $(a, b, c)$ are significantly different one from another (one-way ANOVA followed by Newman-Keuls multiple range test, $\mathrm{P}<0.05)$. $\mathrm{C}$, control rats under regular feeding without $\mathrm{CCl}_{4}$ administration.
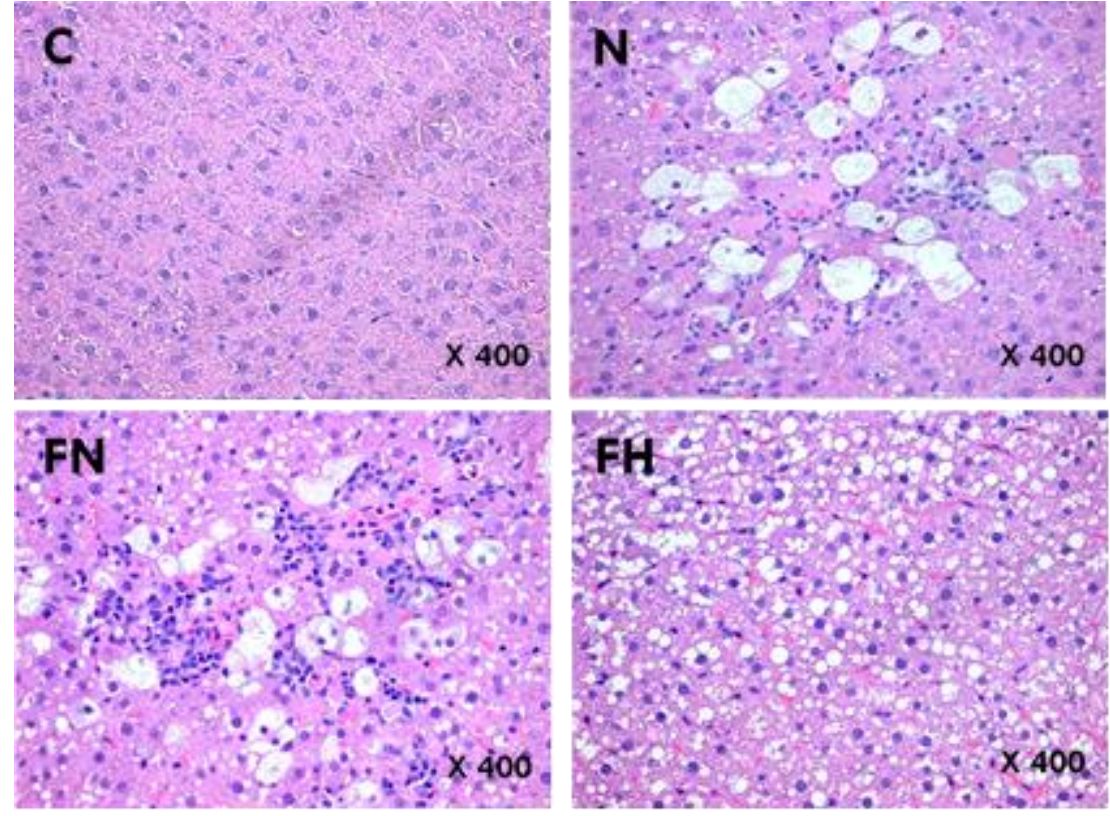

Fig. 5. Histopathological evaluation of liver tissue after $\mathrm{CCl}_{4}$ treatment. Hematoxylin and eosin staining. See the text for details. C, control rats under regular feeding without $\mathrm{CCl}_{4}$ administration.

Numerous studies have shown that hepatic fat accumulation results in significant alterations in the expression and activity of CYP enzymes. However, the results compiled from different studies show a lack of consistency, which could be attributed to a difference in species, sex, and 
most importantly the magnitude or severity of liver steatosis [18]. In general, CYP3A4 expression, as well as its activity, decreased in fatty liver [19,20]. Reduction of other CYPs, including CYP1A2, CYP2C11 and CYP2A1/2, was also demonstrated in association with fat accumulation in liver [21-23]. In contrast, CYP2E1 increased in rodent non-alcoholic steatohepatitis (NASH) models [24], whereas a reduction in CYP2E1 expression was also observed in hepatic steatosis with a lack of inflammation [20]. Therefore, it is speculated that the induction of CYP2E1 in NASH results from complications associated with the inflammatory responses, rather than fat accumulation itself. The present results indicate that CYP enzymes and activities are mostly reduced in acute hepatosteatosis with minimal to no inflammatory reactions.

Treatment of the rats with a dose of $\mathrm{CCl}_{4}$, a CYP2E1 substrate, resulted in an elevation of serum AST, ALT, and SDH activities when determined $24 \mathrm{~h}$ later (Fig. 4). Similar levels of serum enzyme activities were noted in the $\mathrm{FN}$ group challenged with $\mathrm{CCl}_{4}$. However, in the $\mathrm{FH}$ group the elevation of serum enzyme activities was significantly less than that shown in the FN group. Histopathological examination of liver samples showed comparable results (Fig. 5). Marked ballooning degeneration, fatty change, and Councilman bodies were present in hepatocytes of the rats fed FN after fasting and the rats who had regular feeding. There were inflammatory reactions, including migration of neutrophils and infiltration of mononuclear leukocytes. The inflammatory reactions and fatty infiltration in hepatocytes appeared to be more severe in the rats fed FN after fasting than in the rats who had regular feeding. However, there was no infiltration of leukocytes seen in hepatocytes of the rats fed FH after fasting. Additionally, mild ballooning degeneration was present in a few hepatocytes. Meanwhile, lipid peroxidation estimated by TBARS level was not altered by intake of the different diets (data not shown). The results suggest that the reduction of the $\mathrm{CCl}_{4}$ hepatotoxicity is attributable to down-regulation of the CYP2E1 enzyme and its activity associated with hepatic fat accumulation.

\section{CONCLUSIONS}

The present results indicate that refeeding a high carbohydrate/fat-free diet after food deprivation is effective in inducing fatty changes in hepatocyte with minimal or no inflammatory responses. As a result, in the steatotic livers which were induced, expression of the CYP enzymes was markedly reduced. Furthermore, a dose of $\mathrm{CCl}_{4}$ appeared to be less hepatotoxic in the rats fed $\mathrm{FH}$ after fasting than in the rats fed FN after fasting. Therefore, it can be concluded that the prevention of $\mathrm{CCl}_{4}$ toxicity in acute hepatosteatosis is attributed to the inhibition of metabolic 
activation of this solvent, due to the down-regulation of CYP-dependent drug metabolizing enzymes.

List of abbreviations: ALT, alanine aminotransferase; AST, aspartate aminotransferase; CYP, cytochrome P-450; FN, a standard regular diet; FH, high-carbohydrate/fat-free diet; NAFLD, non-alcoholic fatty liver disease; SDH, serum sorbitol dehydrogenase; TBARS, thiobarbituric acid reactive substances.

Competing interests: The authors declare that there are no financial or other conflicts of interest.

Acknowledgements and Funding: This work was supported by National Research Foundation (NRF) grants (No. 2014-R1A2A1A11052967 and No. 2009-0083533) funded by the Ministry of Education, Science and Technology (MEST), Korea.

\section{REFERENCES}

1. Burt AD, Mutton A, Day CP: Diagnosis and interpretation of steatosis and steatohepatitis. Semin Diagn Pathol 1998, 15:246-258.

2. Farrell GC, Larter CZ: Nonalcoholic fatty liver disease: from steatosis to cirrhosis. Hepatology 2006, 43(2 Suppl 1):S99-S112.

3. Yeh MM, Brunt EM: Pathology of nonalcoholic fatty liver disease. Am J Clin Pathol 2007, 128:837-847.

4. Edelman AB, Carlson NE, Cherala G, Munar MY, Stouffer RL, Cameron JL, Stanczyk FZ, Jensen JT: Impact of obesity on oral contraceptive pharmacokinetics and hypothalamic-pituitary-ovarian activity. Contraception 2009, 80:119-127.

5. Skouby SO: Hormonal contraception in obesity, the metabolic syndrome, and diabetes. Ann NY Acad Sci 2010, 1205:240-244.

6. Raucy JL, Kraner JC, Lasker JM: Bioactivation of halogenated hydrocarbons by cytochrome P4502E1. Crit Rev Toxicol 1993, 23:1-20.

7. Avasarala S, Yang L, Sun Y, Leung AW, Chan WY, Cheung WT, Lee SS: A temporal study on the histopathological, biochemical and molecular responses of $\mathrm{CCl}(4)$-induced hepatotoxicity in Cyp2e1-null mice. Toxicology 2006, 228:310-322.

8. Recknagel RO, Glende EA Jr, Dolak JA, Waller RL: Mechanisms of carbon tetrachloride toxicity. Pharmacol Ther 1989, 43:139-154.

9. Plaa GL; Chlorinated methanes and liver injury: highlights of the past 50 years. Annu Rev Pharmacol Toxicol 2000, 40:42-65.

10. Iritani N, Nishimoto N, Katsurada A, Fukuda H: Regulation of hepatic lipogenic enzyme gene expression by diet quantity in rats fed a fat-free, high carbohydrate diet. $J$ Nutr 1992, 122:28-36. 
11. Delzenne NM, Hernaux NA, Taper HS: A new model of acute liver steatosis induced in rats by fasting followed by refeeding a high carbohydrate-fat free diet. J Hepatol 1997, 26: 880-885.

12. Gerlach U: Sorbitol dehydrogenase. In Methods of Enzymatic Analysis. Edited by Bergmeyer HU. Weinheim: Verlag Chemie; 1983:112-117.

13. Reitman S, Frankel SK: A colorimetric method for the determination of serum glutamic oxalacetic and glutamic pyruvic transaminases. Am J Clin Pathol 1957, 28:56-63.

14. Germanò MP, De Pasquale R, D'Angelo V, Catania S, Silvari V, Costa C: Evaluation of extracts and isolated fraction from Capparis spinosa L. buds as an antioxidant source. $J$ Agric Food Chem 2002, 50:1168-1171.

15. Kim YC, Yim HK, Jung YS, Park JH, Kim SY: Hepatic injury induces contrasting response in liver and kidney to chemicals that are metabolically activated: role of male sex hormone. Toxicol Appl Pharmacol 2007, 223:56-65.

16. Kim TS, Freake HC: High carbohydrate diet and starvation regulate lipogenic mRNA in rats in a tissue-specific manner. J Nutr 1996, 126:611-617.

17. Mittendorfer B, Jeschke MG, Wolf SE, Sidossis LS: Nutritional hepatic steatosis and mortality after burn injury in rats. Clin Nutr 1998, 17:293-299.

18. Merrell MD, Cherrington NJ: Drug metabolism alterations in nonalcoholic fatty liver disease. Drug Metab Rev 2011, 43:317-334.

19. Kolwankar D, Vuppalanchi R, Ethell B, Jones DR, Wrighton SA, Hall SD, Chalasani N: Association between nonalcoholic hepatic steatosis and hepatic cytochrome P-450 3A activity. Clin Gastroenterol Hepatol 2007, 5:388-393.

20. Leclercq I, Horsmans Y, Desager JP, Delzenne N, Geubel AP: Reduction in hepatic cytochrome P-450 is correlated to the degree of liver fat content in animal models of steatosis in the absence of inflammation. J Hepatol 1998, 28:410-416.

21. Donato MT, Lahoz A, Jiménez N, Pérez G, Serralta A, Mir J, Castell JV, Gómez-Lechón MJ: Potential impact of steatosis on cytochrome P450 enzymes of human hepatocytes isolated from fatty liver grafts. Drug Metab Dispos 2006, 34:1556-1562.

22. Murray M, Cantrill E, Mehta I, Farrell GC: Impaired expression of microsomal cytochrome P450 2C11 in choline-deficient rat liver during the development of cirrhosis. J Pharmacol Exp Ther 1992, 261:373-380.

23. Su GM, Sefton RM, Murray M: Down-regulation of rat hepatic microsomal cytochromes P-450 in microvesicular steatosis induced by orotic acid. J Pharmacol Exp Ther 1999, 291:953-959.

24. Weltman MD, Farrell GC, Liddle C: Increased hepatocyte CYP2E1 expression in a rat nutritional model of hepatic steatosis with inflammation. Gastroenterology 1996, 111:1645-1653. 